\title{
Ensemble averages, Poisson processes, and microstates
}

\author{
Cheng Peng $\odot^{*}$ \\ Kavli Institute for Theoretical Sciences (KITS) and CAS Center for Excellence in Topological Quantum \\ Computation, University of Chinese Academy of Sciences, Beijing 100190, China \\ and Center for Quantum Mathematics and Physics (QMAP), Department of Physics, \\ University of California, Davis, California 95616, USA
}

(Received 13 January 2021; accepted 17 February 2021; published 22 March 2021)

\begin{abstract}
Ensemble averages of random field theories have recently become promising candidates of the holographic dual of classical gravity. Since quantum theories are discrete, we initiate the study of consistent averages over theories with discrete random variables — which admit a mathematically rigorous description as Poisson processes - and get results that mirror Liouville gravity. We show that this is equivalent to averaging over an ensemble of states in a single microscopic theory, which is an early top-down example trying to answer the crucial question of whether the gravitational path integral computes an ensemble average over true randomness or over pseudorandomness coming from a large number of microstates.
\end{abstract}

DOI: 10.1103/PhysRevD.103.L061901

\section{INTRODUCTION}

Recent studies have uncovered an increasing amount of evidence suggesting that the holographic dual of classical gravity might be an ensemble average of field theories [1-21]. Quantizing an ensemble average of field theories is subtle, even at the conceptual level. In light of this, a general belief about the nature of the ensemble average is that, instead of averaging over true randomness, it is more likely averaged over some microscopic quantum degrees of freedom that display pseudorandomness in appropriate limits. Because the Hilbert spaces of microscopic quantum theories are discrete, the pseudorandomness resulting from those should also take discrete values. Therefore, although by far, the ensemble averages discussed are mostly over Gaussian-type continuous random variables, averaging over discrete random variables could have clearer connections to the microscopic origin. In fact, explicit computations of the gravitational path integral in some simple toy models have demonstrated that their boundary dual descriptions are averages of theories according to discrete probability distributions [22]; see also Refs. [23,24] for related discussions. Given their possible connections to the microscopic discreteness of quantum theories, averages measured by discrete distributions are clearly interesting and worth in-depth studies in their own right.

\footnotetext{
*pengcheng@ucas.ac.cn
}

Published by the American Physical Society under the terms of the Creative Commons Attribution 4.0 International license. Further distribution of this work must maintain attribution to the author(s) and the published article's title, journal citation, and DOI. Funded by SCOAP.
In this paper, we study properties of an average of field theories with random variables drawn from the Poisson distribution. We demonstrate the importance of choosing an appropriate measure for the discrete average to get a wellbehaved effective theory, which in our case is shown to be a Liouville theory. We provide a mathematically more accurate description of such averages in terms of Poisson processes. In this language, the average to get the effective action is nothing but the Laplace functional of the Poisson process.

Moreover, it is interesting to deepen our understanding of the nature of averaging over random theories $[6,8,17$, 25-29] — namely, if the average is genuinely among different theories, or if it is simply a useful trick for certain computations, or if it is originated from averaging among an ensemble of states in a "parent" theory. We study this question quantitatively in our model and show that averages over theories with Poisson randomness are equivalent to a trace over a part of the microscopic degrees of freedom in a single (suitably double-scaled) microscopic model. Such an explicit connection has not been directly manifested in the previous literature; it gives a concrete realization that sets up an equivalence between the average over an ensemble of theories and the average over an ensemble of states in one single theory.

\section{AVERAGED POISSON RANDOM MODELS}

We consider a real scalar field with a chemical potential $J(x)$ in $d$-dimensional Euclidean spacetime:

$$
\begin{aligned}
\mathcal{L}(\phi) & =\partial_{\mu} \phi(x) \partial^{\mu} \phi(x)-J(x) \phi(x), \\
J(x) & =J_{0}(x)+J_{1}(x) .
\end{aligned}
$$


The $J_{0}(x)$ component is a conventional source. The $J_{1}(x)$ component is a random source that we would like to average over. This leads to an effective action, $S_{\text {eff }}$, schematically via

$$
e^{-S_{\text {eff }}}=\int \mathcal{D} J_{1}(x) \mathcal{P}\left(J_{1}(x)\right) e^{-\int d V(x) \mathcal{L}(\phi)},
$$

where $d V(x)$ is a volume element around position $x$. The crucial question is how to pick the correct measure, $\mathcal{D} J_{1}(x) \mathcal{P}\left(J_{1}(x)\right)$. In the following, we give an example of finding such a sensible measure in two different ways, and we will show that they lead to the same results.

\section{A. A physical point of view}

To make the average process well defined, it turns out that we should treat the combination $J_{1}(x) d V(x)$ as the random variable that satisfies

$$
\begin{aligned}
P\left(J_{1}(x), \lambda(x)\right)= & \prod_{n} \operatorname{Pois}\left(J_{1}\left(x_{n}\right) d V\left(x_{n}\right), \lambda\left(x_{n}\right) d V\left(x_{n}\right)\right), \\
& \forall d V\left(x_{n}\right)
\end{aligned}
$$

where Pois $(m, k)=\frac{k^{m}}{m !} e^{-k}$ is the Poisson distribution of the discrete variable $m$ with Poisson parameter $k$. We choose the set $d V\left(x_{n}\right)$ to be a discretization of the spacetime $\sum_{n} d V\left(x_{n}\right)=V(\mathcal{M})$. The sources $J_{1}(x)$ at different positions are mutually independent, so that we can write the probability as a product [Eq. (3)] for any discretization. An example of the configurations to be averaged over is illustrated in Fig. 1.

A straightforward averaging with this probability measure leads to the effective potential

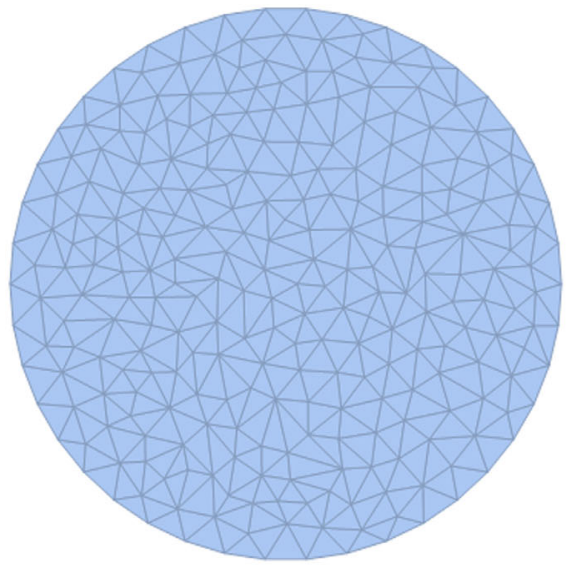

(a)

$$
\begin{aligned}
& \left\langle e^{\int d V(x) J_{1}(x) \phi(x)}\right\rangle_{J_{1}} \\
& :=\int \mathcal{D} J_{1}(x) P\left(J_{1}(x), \lambda(x)\right) e^{\sum_{n} d V\left(x_{n}\right) J_{1}\left(x_{n}\right) \phi\left(x_{n}\right)} \\
& =e^{\int d V(x) \lambda(x)\left(e^{\phi(x)}-1\right)},
\end{aligned}
$$

where the "path integral" $\mathcal{D} J_{1}(x)$ contains both a sum over all the sources at different spacetime points $x$ and a sum over all possible values of $J_{1}(x) d V(x)$. Putting back the kinetic terms, we get the following effective action:

$S_{\mathrm{eff}}=\int d V(x)\left(\partial_{\mu} \phi \partial^{\mu} \phi-J_{0}(x) \phi-\lambda(x)\left(e^{\phi(x)}-1\right)\right)$.

This is a generalized Liouville theory with an effective background curvature $J_{0}(x)$ and a "cosmological constant" term $\lambda(x)$; when $\lambda(x)$ takes a homogeneous value $\lambda(x)=\lambda$, the action reduces to exactly a Liouville action. We will provide a detailed interpretation of this position-dependent expression and its relation to $2 \mathrm{D}$ gravity in Sec. IV.

However, the sign in front of the Liouville potential is "wrong"; the probability interpretation assumes $\lambda(x) \geq 0$, and the minus sign makes the potential unstable in Euclidean signature. To cure this problem, we choose a slightly different measure with an insertion of the $(-1)^{\mathcal{F}}$ "operator":

$$
\begin{aligned}
\left\langle e^{\int}\right. & d V(x) J_{1}(x) \phi(x) \\
& =\int \mathcal{D} J_{1}(x) P\left(J_{1}(x), \lambda(x)\right)(-1)^{\mathcal{F}} e^{\int d V(x) J_{1}(x) \phi(x)} \\
& =e^{\int d V(x) \lambda(x)\left(-e^{\phi(x)}+1\right)},
\end{aligned}
$$

where

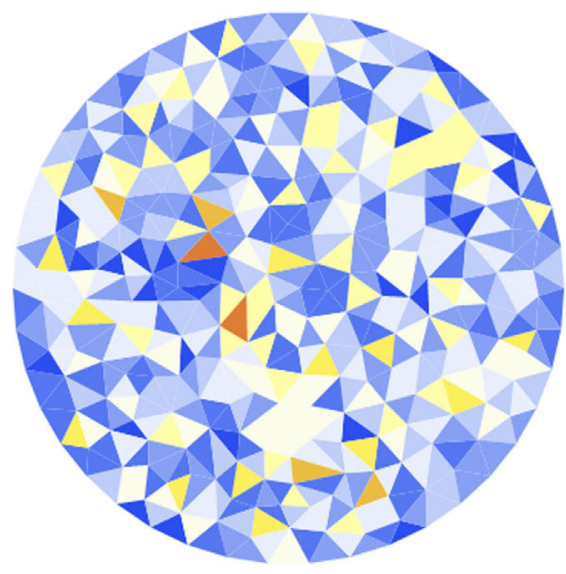

(b)

FIG. 1. An illustration of the random configurations discussed around Eq. (3). Different colors on the right figure represent different values of the fluxes $J_{1}(x) d V(x)$; the color changes from blue to red representing the fluxes changing from 0 to $\infty$. (a) An example of discretizations of the spacetime. (b) An illustration of the quantized "fluxes" on discretization of the spacetime. 


$$
(-1)^{\mathcal{F}} \equiv(-1)^{\int J_{1}(x) d V(x)} e^{2 \int d V(x) \lambda(x)}
$$

and the $J_{1}$ independent factor is introduced so that the average is correctly normalized:

$$
\begin{aligned}
& \int \mathcal{D} J_{1}(x) P\left(J_{1}(x), \lambda(x)\right)(-1)^{\mathcal{F}} \\
& \equiv \int \mathcal{D} J_{1}(x) \mathcal{P}\left(J_{1}(x), d V(x)\right)=1 .
\end{aligned}
$$

Since there is no randomness in the other terms, with this twist operator inserted we obtain the following effective action:

$$
S_{\mathrm{eff}}=\int d V(x)\left(\partial_{\mu} \phi \partial^{\mu} \phi-J_{0}(x) \phi+\lambda(x)\left(e^{\phi(x)}-1\right)\right) .
$$

In summary, requiring the resulting theory to be well defined, we found a proper measure for the average of the set of theories [Eq. (1)] that necessarily includes a "twist" factor [Eq. (7)]. As in many other recent discussions of ensemble averages of theories $[10,11]$, at the current stage we do not have a general criterion to determine what family of theories should be averaged over, and with what measure. Our discussion provides an early nontrivial example that answers these questions.

In fact, this twist factor resembles the $(-1)^{F}$ factor of the Witten index [30] in supersymmetric theories. The presence of this factor gives a clear indication, together with the fact that our probability distribution is discrete, that there should be a microscopic origin of this random averaged model; and in addition, there must be fermionic degrees of freedom in the microscopic model so that an invariant counting of the states, which requires the $(-1)^{F}$ insertion, leads to the macroscopic averaging. This is confirmed in Sec. III.

\section{B. A Poisson process point of view}

The computation in the above section admits a mathematically more rigorous, yet still intuitive, formulation. The crucial observation is that we can interpret the source $J_{1}(x)$ as a Poisson process on the carrier space $X=R^{d}$.

A Poisson process $\Pi$ describes a random set of points on a given carrier space whose probability of [the points in the set] appearing obeys a Poisson distribution [31]. Concretely, let $B$ be a Borel measurable subset of the carrier space $X$. Let the number of the points of $\Pi$ appearing in this region $B$ be $N(B) \equiv \#(\Pi \cap B)$. Then $N(B)$ on disjoint open subsets $B_{1}, \ldots, B_{n}$ is mutually independent and satisfies the Poisson distribution

$$
P(N(B)=n)=\frac{\Lambda(B)^{n}}{n !} e^{-\Lambda(B)},
$$

where the Poisson parameter $\Lambda(B)$ is also the mean value $\mathbb{E}[N(B)]=\Lambda(B)$ and is determined by a given integrable function $\lambda(x)$-i.e., the intensity, via $\Lambda(B)=\int_{B} \lambda(x) d V(x)$. For infinitesimal $B$, the integral is well approximated by

$$
\Lambda(d x)=\lambda(x) d V(x) .
$$

The $\Lambda(x)$ or $\lambda(x)$ functions describe the "shape" of the Poisson distributions on the carrier space. A numerical simulation of a Poisson process is shown in Fig. 2.

In our setting, the Poisson random variable is

$$
\int_{B} d V(x) J_{1}(x) \sim N(B) .
$$

For any open set, whose scale is small enough compared with the scale at which the field $\phi$ varies, we can safely approximate

$$
N(d x) \sim J_{1}(x) d V(x):=\mathcal{J}(d x) \in \mathbb{Z}_{\geq 0} .
$$

Averaging over these random sources is, in this language, nothing but the Laplace functional:

$\mathbb{E}\left[e^{\int d V(x) J_{1}(x) \phi(x)}\right]=\mathbb{E}\left[e^{\int \mathcal{J}(d x) \phi(x)}\right]=e^{\int_{\mathcal{M}} \Lambda(d x)\left(e^{\phi}(x)-1\right)}$.

This resulting Liouville potential is again unbounded from below. To cure this, we can similarly insert a "twist"

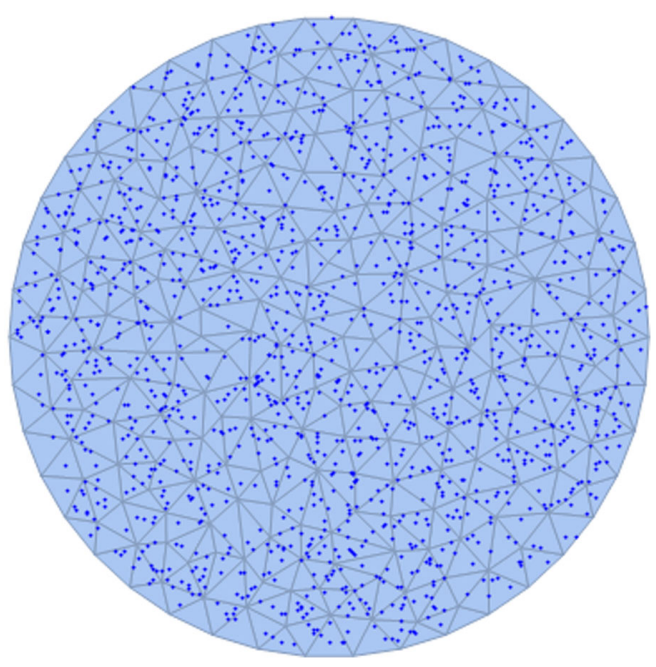

FIG. 2. A numerical simulation of a Poisson process. The blue dots represent the Poisson process on a two-dimensional carrier space (the light-blue background). The background is the same random discretization of the carrier space as shown in Fig. 1, and each element of the Borel subset can be chosen as a union of cells in this discretization. In this example, the intensity measure is a constant, $\lambda(x)=1$, which represents a homogeneous Poisson process. The intersection number $N(B)$ on each cell can be identified with the quantized fluxes in each cell shown in Fig. 1(b). 
factor $(-1)^{\int \mathcal{J}(d x)}$ in computing the expectation value. Alternatively, an equivalent effect can be achieved by considering a slightly modified point process with an "alternating" Poisson distribution

$$
P(N(B)=n)=\frac{(-\Lambda(B))^{n}}{n !} e^{\Lambda(B)} .
$$

Although the extra sign could make the classical probability interpretation of the $P$ function obscure, it is perfectly compatible with the definition of the point process, in particular, it is compatible with the requirement of independence among different spatial regions. So we can simply consider it as a different measure defining a new point process, with which the Laplace functional that computes the effective potential becomes

$\tilde{\mathbb{E}}\left[e^{\int d^{d} x J_{1}(x) \phi(x)}\right]=\tilde{\mathbb{E}}\left[e^{\int \mathcal{J}(d x) \phi(x)}\right]=e^{-\int_{\mathcal{M}} \Lambda(d x)\left(e^{\phi}(x)-1\right)}$.

Adding back the kinetic term, we again arrive at the same effective action in Eq. (9), now from the viewpoint of Poisson processes.

\section{POISSON RANDOM AVERAGE FROM TRACING OVER MICROSTATES}

Conventional quantization is done for a given theory with a fixed action, which could be an obstruction to further understanding ensemble averaged theories, and in particular their quantum counterparts. A way out is to consider the ensemble averaged theory as an effective description of the low-energy limit of (a subsystem of) a microscopic theory. In this section, we materialize this idea into an explicit connection that reformulates the above average over the Poisson random potentials into a trace over a large number of microscopic degrees of freedom in a single refined model.

\section{A. The microscopic setting}

We consider a model defined on a (microscopic) spatial lattice, labeled by a "position" vector $x$, on each site of which resides a $d$-level system with a Hilbert space $\mathcal{H}_{x}$ spanned by state vectors $|i\rangle_{x}, i=1, \ldots, d\left(\mathcal{H}_{x}\right) \equiv d_{x}$. One simple example is a theory of $N$ pairs of free complex fermions $\psi^{i}, \bar{\psi}^{i}, i=1, \ldots, N$ on each site, so that the dimension of the Hilbert space is $d_{x}=2^{N}$. We can choose the ground state $|0\rangle$ to be annihilated by $\psi^{i}$ so that the states in the Hilbert space are spanned by $\bar{\psi}^{i_{1}} \ldots \bar{\psi}^{i_{k}}|0\rangle$. The simplest example is $N=1$, with states $|0\rangle_{x}$ and $|1\rangle_{x}$. We further introduce a free boson $\phi_{x}$ at each lattice site. The system is described by the Hamiltonian

$$
\begin{aligned}
H & =\sum_{x} H_{x, 0}+H_{x, 1}, \\
H_{x, 0} & =\pi_{x}^{2}+\frac{m}{2} \phi_{x}^{2}+\sum_{y} t_{x y} \phi_{x} \phi_{y}+J_{0}(x) \phi_{x}, \\
H_{x, 1} & =m \bar{\psi}_{x} \psi_{x}-\bar{\psi}_{x} \psi_{x} \phi_{x},
\end{aligned}
$$

where $\pi_{x}$ is the conjugate momentum of $\phi_{x}$. We prepare the system to be in a mixed state whose density matrix factorizes

$$
\begin{aligned}
\rho & =\rho_{\phi} \otimes \rho_{\psi}, \quad \rho_{\psi}=\underset{x}{\otimes} \rho_{x}, \\
\rho_{x} & =(1-p(x))|0\rangle_{x}\langle 0|+p(x)| 1\rangle_{x}\langle 1| .
\end{aligned}
$$

We would like to trace over the fermionic components, and run into low energy to get an effective dynamics for the bosons.

\section{B. Tracing over fermionic states}

We trace over the fermions to get an effective Hamiltonian $H_{\text {eff }}$ according to

$$
\begin{aligned}
e^{-\beta H_{\text {eff }}} & =\operatorname{Tr}_{\mathcal{H}_{\psi}}\left((-1)^{F} \rho e^{-\beta H}\right)=\operatorname{Tr}_{\mathcal{H}_{\psi}}\left(\rho e^{-\beta H+i \pi F}\right) \\
& :=\operatorname{STr}_{\mathcal{H}_{\psi}}\left(\rho e^{-\beta H}\right) .
\end{aligned}
$$

This choice is invariant under a change of basis of the fermionic degrees of freedom. Alternatively, this choice effectively turns on an imaginary chemical potential of the fermion number operator, or equivalently imposes a Ramond boundary condition for the fermions.

We consider the simplest case on an infinite square lattice with lattice parameter $a$. We consider a "continuum limit" parametrized by $a=\frac{1}{m}$ with $m \rightarrow \infty$. It is clear that these grid points are bijective to the grid of rational points. It is also clear that any open set on the background $\mathcal{M}=\mathbb{R}^{d}$ contains an infinite number of grid points with a density $n=m^{d}$ in the above $m \rightarrow \infty$ limit, which is the familiar fact that in any open set of $\mathbb{R}$ there are countably infinite rational points. This fact remains true even if the volume of the open set is infinitesimal, such as the volume element $d V(x)$.

We assume that $p(x)$ in Eq. (18) is smooth enough so that in any small region $d V(x)$ it is approximately constant. The probability to have $n_{x}=k$ fermionic excitations in this volume is

$$
P\left(n_{x}=k\right)=\left(\begin{array}{c}
n^{\prime} \\
k
\end{array}\right) p(x)^{k}(1-p(x))^{n^{\prime}-k},
$$

where $n^{\prime}=n d V(x)$ is the total number of grid points in the volume element and $n_{x}$ is the total number of sites that are in the $|1\rangle$ states in $d V(x)$. A continuum limit can be appropriately defined if the number density of microscopic excitations in the fermion systems is finite: 


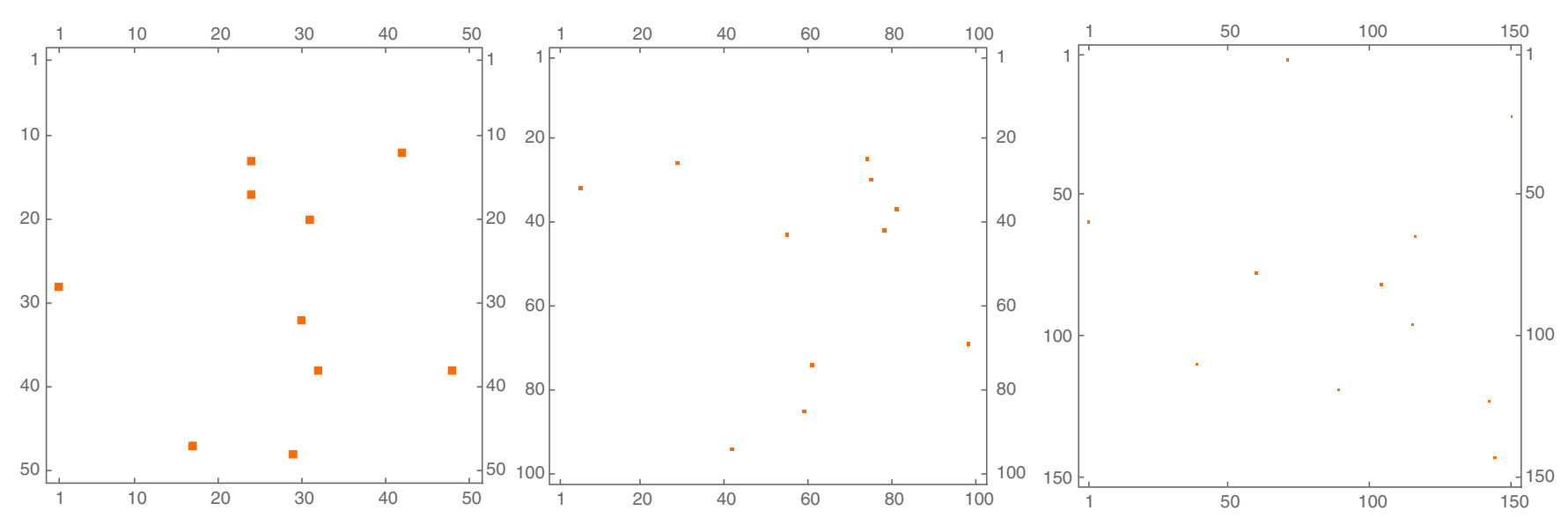

FIG. 3. A numerical illustration of the limit [Eq. (21)]. The red dots are positions on the lattice where the state is in the $|1\rangle$ state. From left to right, we have $n^{\prime}=2.5 \times 10^{3}, 10^{4}, 2.25 \times 10^{4}$, respectively, while $\lambda(x)$ is fixed to 1 [with $d V(x)=10$ to make the figures easier to read]. It is clear that as $n^{\prime} \rightarrow \infty$, the expected number of excited states is approximately fixed.

$\lim _{n^{\prime} \rightarrow \infty} n^{\prime} p(x)=\Lambda_{d x}(x)=\lambda(x) d V(x), \quad \lambda(x) \sim \mathcal{O}(1)$

as illustrated in Fig. 3.

In this limit, the probability of having $n_{x}=k$ excited $|1\rangle$ states in the volume $d V(x)$ becomes

$$
\begin{aligned}
\lim _{n^{\prime} \rightarrow \infty} P\left(n_{x}=k\right) & =\frac{n^{\prime} !}{k !\left(n^{\prime}-k\right) ! n^{\prime k}}\left(n^{\prime} p\right)^{k}(1-p)^{n^{\prime}-k} \\
& =\frac{\Lambda_{d x}(x)^{k}}{k !} e^{-\Lambda_{d x}(x)}=\operatorname{Pois}\left(k, \Lambda_{d x}(x)\right),
\end{aligned}
$$

which means in the limit (21), the distribution of excitations obey the Poisson distribution on any open set $d V(x)$. We further consider a semiclassical limit where $\phi_{x}$ varies slowly on the microscopic scale so that in each $d V(x)$ it can be regarded as a constant. Then the $H_{x, 1}$ contribution to the trace [Eq. (19)] becomes

$$
\begin{aligned}
e^{-\beta H_{\text {eff }}} & =\operatorname{Tr}_{\mathcal{H}_{\psi}}\left(\rho e^{-\beta H+i \pi F}\right) \\
& =\operatorname{Tr}_{\mathcal{H}_{\psi}}\left(\bigotimes_{d V(x)} \rho_{x} e^{-\beta \sum_{d V(x)}\left(\sum_{x \in d V(x)}\left(H_{x}-\frac{i \pi}{\beta} F F_{d x}\right)\right)}\right) \\
& =\prod_{d V(x)}\left(\sum_{k} \operatorname{Pois}\left(\Lambda_{d x}(x), k\right) e^{-\beta\left(k\left(m-\phi_{x}-\frac{i \pi}{\beta}\right)\right)}\right) \\
& =e^{-\beta \int \frac{\lambda(x)}{\beta} d x\left(e^{-\beta\left(m-\phi_{x}\right)}+1\right)} .
\end{aligned}
$$

Therefore, we get an effective Hamiltonian

$$
\begin{aligned}
\mathcal{H}(x) & =\frac{\lambda(x)}{\beta} e^{-\beta\left(m-\phi_{x}\right)}+\frac{\lambda(x)}{\beta} \\
& =\frac{\lambda(x)}{\beta} e^{-\beta m} e^{\beta \phi_{x}}+\frac{\lambda(x)}{\beta} \\
& :=2 \pi \mu(x) e^{2 b \phi_{x}}+\frac{\lambda(x)}{2 b},
\end{aligned}
$$

where

$$
b=\frac{\beta}{2}, \quad \mu=\frac{\lambda}{2 \pi \beta} e^{-\beta m}=\frac{\lambda}{4 \pi b} e^{-2 b m},
$$

and it is similar with the (generalized) Liouville potential in Eqs. (4) and (16). Putting back the pure bosonic contribution $H_{x, 0}$ that is independent of the trace, we get

$$
\begin{aligned}
\mathcal{H}(x)= & \pi_{x}^{2}+\frac{m}{2} \phi_{x}^{2}+\sum_{y} t_{x y} \phi_{x} \phi_{y}+J_{0}(x) \phi(x) \\
& +2 \pi \mu(x) e^{2 b \phi_{x}}+\frac{\lambda(x)}{2 b} .
\end{aligned}
$$

To summarize, in the special scaling limit (21), the effective interaction Hamiltonian (23) is proportional to $n_{x}$, which is mutually independent in each subset $d V(x)$ due to the absence of fermionic hopping terms. The probability of $n_{x}$ in the limit (21) satisfies a Poisson distribution [Eq. (20)]. These together mean that the limit can be described by a Poisson process with $n_{x}$ analogous to the random variable [Eq. (10)] and $n^{\prime} p(x), n p(x)$ analogous to the mean measure and the intensity. Therefore, as discussed in Sec. II B, Eq. (23) can be considered as an analogue of an ensemble of theories with a source $N_{x}$ that is related to a discrete Poisson distribution that is of the same type of the theory defined by Eq. (1). This demonstrates explicitly that an ensemble average of theories could actually be equivalently an average of an ensemble of states in a single theory.

\section{The low-energy limit}

Next, we focus on the low-energy modes which are expected to have a canonical kinetic term in continuous spacetime and are exactly described by a Liouville theory. 
This amounts to going to the frequency space and extracting the effective action near zero momentum.

For simplicity, we consider the simplest $(1+1)$-dimension case. We further simplify the computation by considering a homogeneous chain with the nearest-neighbor hopping, namely $|x-y|=1$

$$
t_{x y}=t_{y x}=\frac{1}{2} t \delta(|x-y|-1) .
$$

We can now take the familiar route of Fourier-transforming to frequency space and reading out the effective Hamiltonian for the low-energy modes,

$$
\begin{aligned}
H= & \sum_{x}\left(\pi_{x}^{2}+\frac{1}{c^{2}}\left(\partial_{x} \phi(x)\right)^{2}+m_{\phi}^{2} \phi(x)^{2}-J_{0}(x) \phi(x)\right. \\
& \left.+2 \pi \mu(x) e^{2 b \phi(x)}+\frac{\lambda(x)}{2 b}\right)
\end{aligned}
$$

where

$m=2 m_{\phi}^{2}+2 t, \quad t=\frac{2}{c^{2}} a^{-2} \geq 0, \quad m_{\phi}, a, c \in \mathbb{R}$.

The result in Eq. (28) is again a generalized Liouville theory.

\section{DISCUSSION}

\section{A. Relation to Gaussian randomness}

This work highlights discrete distributions. It is clear that a further limit of the Poisson distribution approaches a Gaussian distribution, which is guaranteed by the central limit theorem. Therefore, we can also regard the discussion in this paper as a first attempt towards refining the results in previous Gaussian random (holographic) discussions.

\section{B. The dual channel}

We can also integrate out the $\phi_{x}$ field in Eq. (17) to get a $0+1 \mathrm{D}$ quantum mechanical model of the fermions. It is easier to work in the Lagrangian formalism, where the coupled theory is defined as

$$
\begin{aligned}
L= & \sum_{x}\left(\frac{1}{2} \dot{\phi}_{x}^{2}+\frac{m_{\phi}}{2} \phi_{x}^{2}+\frac{t_{x-1, x}}{2} \phi_{x-1} \phi_{x}+\frac{t_{x, x+1}}{2} \phi_{x} \phi_{x+1}\right. \\
& \left.+J_{0}(x) \phi_{x}+i \bar{\psi} \dot{\psi}+m \bar{\psi}_{x} \psi_{x}-\bar{\psi}_{x} \psi_{x} \phi_{x}\right) .
\end{aligned}
$$

We can integrate out the $\phi_{x}$ to get

$$
e^{-\int d t L_{\text {eff }}}=\int \mathcal{D} \phi_{x} e^{-\int d t L} \sim e^{-\frac{1}{2} \log (M)+\int d t\left(i \sum_{x} \bar{\psi}_{x} \dot{\psi}_{X}+m \sum_{x} \bar{\psi}_{x} \psi_{x}+\frac{1}{2} \sum_{x, y} \bar{\psi}_{x} \psi_{x} \bar{\psi}_{y} \psi_{y}\left(M^{-1}\right)_{x y}\right)}
$$

where " " means up to irrelevant constants, and the matrix $M$ is defined to be

$$
M_{x y}=-\partial_{\tau}^{2} \delta_{x, y}+m_{\phi} \delta_{x, y}+t_{x-1, x} \delta_{y, x-1}+t_{x, x+1} \delta_{y, x+1} .
$$

So the dual quantum mechanical model is described by a "nearest neighbor" charge-charge coupling.

In addition, the coupling constant $M^{-1}$ has a singularity if the $\phi$ field is gapless in the infrared, namely $m_{\phi}=0$ in Eq. (29); this is consistent with our expectation of integrating out a massless mode.

\section{Interpretation as emergent gravity?}

Recall that Liouville gravity, which can be obtained from the response of the matter field to a Weyl transformation of the metric $h_{\mu \nu} \rightarrow e^{\phi} h_{\mu \nu}$ it couples to, has the action

$$
I_{\mathrm{L}}=\frac{1}{4 \pi} \int \mathrm{d}^{2} x \sqrt{|h|}\left(Q \Phi(x) R_{h}(x)+(\nabla \Phi)^{2}+4 \pi \mu e^{2 b \Phi(x)}\right),
$$

where $\mu$ is the cosmological constant and $Q$ is a background charge related to the property, such as the central charge, of the matter fields [32].
Comparing with our results, we find the probabilistic measure $\lambda(x)$ actually resembles the density $\sqrt{|h|}$ in gravity. In fact, we can directly show the equivalence of the actions by identifying the dilaton $\Phi(x)$ in Eq. (33) and the scalar $\phi(x)$ in Eq. (9) together with the following matching:

$Q \sqrt{|h|} R_{h}(x)=-J_{0}(x), \quad 4 \pi \mu \sqrt{|h|}=2 \pi \mu(x)=\frac{\lambda(x)}{2 b} e^{-2 m b}$,

$\sqrt{|h|} h^{\mu \nu} \partial_{\mu} \partial_{\nu}=\delta^{\mu \nu} \partial_{\mu} \partial_{\nu}$.

In order to make the comparison transparent, we fix a gauge in the Liouville gravity $h_{\mu \nu}=e^{\rho(x)} \delta_{\mu \nu}$; then the correspondence reduces to

$$
\begin{aligned}
-Q \delta^{\mu \nu} \partial_{\mu} \partial_{\nu} \rho(x) & =-J_{0}(x), \\
4 \pi \mu e^{\rho(x)} & =2 \pi \mu(x)=\frac{\lambda(x)}{2 b} e^{-2 m b}, \\
\delta^{\mu \nu} \partial_{\mu} \partial_{\nu} & =\delta^{\mu \nu} \partial_{\mu} \partial_{\nu} .
\end{aligned}
$$

The third equality trivializes, while the first two identify $\lambda(x)=8 \pi \mu b e^{\rho(x)+2 m b}$, and further imply a condition between $J_{0}(x)$ and $\lambda(x)$ : 


$$
\frac{J_{0}(x)}{Q}=\delta^{\mu \nu} \partial_{\mu} \partial_{\nu} \log \left(\frac{\lambda(x) e^{-2 m b}}{8 \pi \mu b}\right)=\delta^{\mu \nu} \partial_{\mu} \partial_{\nu} \log (\lambda(x)) .
$$

This is a very interesting relation that furnishes a necessary condition for the random or microscopic theory to have a gravity description. In particular, it states that $J_{0}$, which is initially introduced as the source of the $\phi(x)$ field, is also the source of $\log (\lambda(x))$ if we regard the latter as a free classical field. On the other hand, the limit (21) and the condition (36) cannot isolate any single state in the microscopic theory; a large number of states are not distinguishable in the above semiclassical limit where a gravity interpretation applies. It is likely that these different states that are compatible with Eq. (21) carry information about quantum corrections to the classical gravity, similar to the recent discussion in terms of the JT/random matrix model example [35].

Further notice that Eq. (36) only fixes the shape dependence; the size of the $J_{0}(x)$ field can, however, vary, and it is proportional to $Q$. In the gravity interpretation, $Q$ roughly counts the number of degrees of freedom of the matter fields, and hence indicates the strength of their coupling to gravity. Therefore, the scale of $J_{0}(x)$ in our microscopic model could be understood as the strength of the coupling of $\phi(x)$ to some other matter fields as expected.

\section{ACKNOWLEDGMENTS}

The author is grateful to Zhixian Zhu and many members of QMAP and KITS for interesting discussions. This work is supported in part by the U.S. Department of Energy Grant No. DE-SC0019480 under the HEP-QIS QuantISED program and by funds from the University of California. After the author leaves the US and moves to the current institute, he starts to receive funds from the Kavli Institute for Theoretical Science (KITS) and a startup funding from the University of Chinese Academy of Sciences (UCAS).
[1] J. Maldacena and D. Stanford, Remarks on the Sachdev-YeKitaev model, Phys. Rev. D 94, 106002 (2016).

[2] J. Maldacena, D. Stanford, and Z. Yang, Conformal symmetry and its breaking in two dimensional nearly anti-de Sitter space, Prog. Theor. Exp. Phys. 2016, 12 C104 (2016).

[3] J. Maldacena, D. Stanford, and Z. Yang, Diving into traversable wormholes, Fortschr. Phys. 65, 1700034 (2017).

[4] J. S. Cotler, G. Gur-Ari, M. Hanada, J. Polchinski, P. Saad, S. H. Shenker, D. Stanford, A. Streicher, and M. Tezuka, Black holes and random matrices, J. High Energy Phys. 05 (2017) 118.

[5] P. Saad, S. H. Shenker, and D. Stanford, A semiclassical ramp in SYK and in gravity, arXiv:1806.06840.

[6] P. Saad, S. H. Shenker, and D. Stanford, JT gravity as a matrix integral, arXiv:1903.11115.

[7] D. Stanford and E. Witten, JT gravity and the ensembles of random matrix theory, arXiv:1907.03363.

[8] G. Penington, S. H. Shenker, D. Stanford, and Z. Yang, Replica wormholes and the black hole interior, arXiv: 1911.11977.

[9] E. Witten, Matrix models and deformations of JT gravity, Proc. R. Soc. A 476, 20200582 (2020).

[10] N. Afkhami-Jeddi, H. Cohn, T. Hartman, and A. Tajdini, Free partition functions and an averaged holographic duality, J. High Energy Phys. 01 (2021) 130.

[11] A. Maloney and E. Witten, Averaging over Narain moduli space, J. High Energy Phys. 10 (2020) 187.

[12] A. Belin and J. de Boer, Random statistics of OPE coefficients and Euclidean wormholes, arXiv:2006.05499.

[13] A. Pérez and R. Troncoso, Gravitational dual of averaged free CFT's over the Narain lattice, J. High Energy Phys. 11 (2020) 015.
[14] J. Cotler and K. Jensen, $\mathrm{AdS}_{3}$ gravity and random CFT, arXiv:2006.08648.

[15] H. Maxfield and G. J. Turiaci, The path integral of 3D gravity near extremality; or, JT gravity with defects as a matrix integral, J. High Energy Phys. 01 (2021) 118 .

[16] A. Blommaert, Dissecting the ensemble in JT gravity, arXiv:2006.13971.

[17] R. Bousso and E. Wildenhain, Gravity/ensemble duality, Phys. Rev. D 102, 066005 (2020).

[18] M. Van Raamsdonk, Comments on wormholes, ensembles, and cosmology, arXiv:2008.02259.

[19] D. Stanford, More quantum noise from wormholes, arXiv: 2008.08570.

[20] C. V. Johnson, Low energy thermodynamics of JT gravity and supergravity, arXiv:2008.13120.

[21] D. Marolf and H. Maxfield, Observations of Hawking radiation: The Page curve and baby universes, arXiv: 2010.06602.

[22] D. Marolf and H. Maxfield, Transcending the ensemble: Baby universes, spacetime wormholes, and the order and disorder of black hole information, J. High Energy Phys. 08 (2020) 044.

[23] T. Anous, J. Kruthof, and R. Mahajan, Density matrices in quantum gravity, SciPost Phys. 9, 045 (2020).

[24] V. Balasubramanian, A. Kar, S. F. Ross, and T. Ugajin, Spin structures and baby universes, J. High Energy Phys. 09 (2020) 192.

[25] A. Almheiri, N. Engelhardt, D. Marolf, and H. Maxfield, The entropy of bulk quantum fields and the entanglement wedge of an evaporating black hole, J. High Energy Phys. 12 (2019) 063. 
[26] A. Almheiri, T. Hartman, J. Maldacena, E. Shaghoulian, and A. Tajdini, Replica wormholes and the entropy of Hawking radiation, J. High Energy Phys. 05 (2020) 013.

[27] J. McNamara and C. Vafa, Baby universes, holography, and the swampland, arXiv:2004.06738.

[28] J. Pollack, M. Rozali, J. Sully, and D. Wakeham, Eigenstate Thermalization and Disorder Averaging in Gravity, Phys. Rev. Lett. 125, 021601 (2020).

[29] K. Langhoff and Y. Nomura, Ensemble from coarse graining: Reconstructing the interior of an evaporating black hole, Phys. Rev. D 102, 086021 (2020).

[30] E. Witten, Constraints on supersymmetry breaking, Nucl. Phys. B202, 253 (1982).
[31] J. Kingman, Poisson Processes, Oxford Studies in Probability (Clarendon Press, Oxford, 1992).

[32] See, e.g., Refs. [15,33,34] for some recent discussions about the connection between Liouville theory and random matrix models/JT gravity.

[33] D. Bagrets, A. Altland, and A. Kamenev, SachdevYe-Kitaev model as Liouville quantum mechanics, Nucl. Phys. B911, 191 (2016).

[34] T. G. Mertens and G. J. Turiaci, Liouville quantum gravity: Holography, JT and matrices, J. High Energy Phys. 01 (2021) 073.

[35] P. Saad, Late time correlation functions, baby universes, and ETH in JT gravity, arXiv:1910.10311. 